\title{
Vanishing of Cohomology Groups on Completely $k$-Convex Sets
}

\author{
By \\ Takahiro KAWAI*
}

The purpose of this paper is to prove, for a completely $k$-convex open (compact, respectively) set, the vanishing of cohomology groups having as their coefficients the hyperfunction (real analytic, respectively) solution sheaf of linear differential equations with constant coefficients (Theorem 1 and Theorem 2, respectively). We also discuss the vanishing theorem for relative cohomology groups (Theorem 3) and the vanishing theorem for completely $k$-concave open sets (Definition 3 and Theorem 4). A theorem on the extendability of the solutions is naturally obtained in the course of the proof (Theorem 5). This is a natural generalization of the celcbrated theorem of Ehrenpreis [2] on removable singularity of solutions of a system of linear differential equations with constant coefficicnts. The main result of this paper, i.c. Theorem 1, has been announced in Kawai [5]. The notion of completely $k$-convex open set was first introduced by Palamodov [9] under the influence of AndreottiGrauert [1].

In this paper we denote by $A$ the ring $\mathbb{C}\left[\xi_{1}, \ldots, \xi_{n}\right]$ of polynomials in $n$ indeterminates $\xi_{1}, \ldots, \xi_{n}$ with coefficients in $\mathbb{C}$.

Let us first recall the definition of completely $k$-convex open set $\Omega \subset \mathbf{R}^{n}$.

Definition 1. (Palamodov [9] Chapter VII §11) An open set $\Omega$ $\subset \mathbb{R}^{n}$ is said to be completely $k$-convex if and only if there exists a $C^{2}$-function $h(x)$ defined on $\Omega$ which satisfies the following two conditions:

Received June 17, 1975.

* Work done while visiting Department of Mathematics, University of California, Berkclcy, U.S. A., and supportcd by Miller Institute for Basic Rescarch in Science. 
(1) Hess $h(x)=\left(\frac{\partial^{2} h}{\partial x_{i} \partial x_{j}}\right)_{1 \leqq i, j \leqq n}$ has at least $k$ positive eigenvalues.

(2) The set $K_{c}=\{x \in \Omega ; h(x) \leqq c\}$ is compact for any real number $c$.

Remark. We call $h(x)$ a norm function associated with $\Omega$.

Theorem 1. Let an open set $\Omega \subset \mathbb{R}^{n}$ be completely $(n-k)$-convex. Then, for any system $M$ of linear differential equations with constant coefficients defined on $\mathbb{R}^{n}$, we have

$$
\operatorname{Ext}^{i}(M, \mathscr{B}(\Omega))=0
$$

for $i>k$. Here $\mathscr{B}(\Omega)$ denotes the space of hyperfunctions defined on $\Omega$.

Proof. Let $I, I^{+}$and $I^{-}$be open intervals in $\mathbb{R}$ given by $(-1,1)$, $(0,1)$ and $(-1,0)$, respectively. It is clear that $\Omega \times I \underbrace{\times \cdots \times I}_{j}$ and $\Omega$ $\times I \underbrace{\times \cdots \times}_{j-1} \times I \times I^{ \pm}$are completely $(n-k+j)$-convex. In the sequel we denote $\Omega \times I \underbrace{\times \cdots \times}_{j} I$ and $\Omega \times \underbrace{I \times \cdots \times}_{j-1} \times I \times I^{ \pm}$by $\tilde{\Omega}_{j}$ and $\tilde{\Omega}_{j}^{ \pm}$, respectively. In fact, if we define $h_{j}\left(x, t_{1}, \ldots, t_{j}\right)$ by $h(x)+\sum_{i=1}^{j} \frac{1}{1-t_{i}^{2}}$, its Hessian matrix has at least $(n-k+j)$ positive eigenvalues on $\widetilde{\Omega}_{j}$. Further, the set $\tilde{K}_{c}=\left\{(x, t) \in \widetilde{\Omega}_{j} ; h_{j}(x, t) \leqq c\right\}$ is compact for any $c \in \mathbb{R}$, since $K_{c}=\{x$ $\in \Omega ; h(x) \leqq c\}$ is compact. Thus $\widetilde{\Omega}_{j}$ turns out to be completely $(n-k+j)$ convex with norm function $h_{j}(x, t)$. It is clear that the same is ture for $\tilde{\Omega}_{j}^{ \pm}$.

Now we define system $\tilde{M}_{n}$ of linear differential equations defined on $\mathbb{R}^{2 n}$ by making the tensor product of $M$ and the Cauchy-Riemann equation $L:\left(\frac{\partial}{\partial x_{j}}+\sqrt{-1} \frac{\partial}{\partial t_{j}}\right) u(x, t)=0(j=1, \ldots, n)$ on $\mathbb{R}^{2 n} \cong \mathbb{C}^{n}$. Define $\tilde{M}_{j}$ by the tangential system of linear differential equations of $\tilde{M}_{n}$ induced onto $\mathbb{R}^{n} \times \mathbb{R}^{j} \times\{0\} \times \cdots \times\{0\} \subset \mathbb{R}^{2 n}$. See Sato-Kawai-Kashiwara [11] Chapter II Definition 3.5.4 ${ }^{n-j}$ for the definition of tangential systems. Note that $\mathbb{R}^{n} \times \mathbb{R}^{j} \times\{\underbrace{0\} \times \cdots \times\{0\}}_{n-j}$ is non-characteristic with respect to $\tilde{M}_{n}$. Clearly $\tilde{M}_{0}=M$.

Since sheaf $\mathscr{B}$ of hyperfunctions is flabby (Sato [10]), we have the following long exact sequences of relative cohomology groups: 
(4)

$$
\begin{aligned}
\cdots & \longrightarrow \operatorname{Ext}^{i}\left(\tilde{M}_{j+1}, \mathscr{B} \widetilde{\Omega}_{j} \times\{0\}\right. \\
& \left.\left.\longrightarrow \tilde{\Omega}_{j+1}\right)\right) \longrightarrow \operatorname{Ext}^{i}\left(\tilde{M}_{j+1}, \mathscr{B}\left(\widetilde{\Omega}_{j+1}-\tilde{\Omega}_{j} \times\{0\}\right)\right) \\
& \left.\longrightarrow \tilde{M}_{j+1}, \mathscr{B}\left(\tilde{\Omega}_{j+1}\right)\right) \\
& \tilde{E x}^{i+1}\left(\tilde{M}_{j+1}, \mathscr{B}_{\widetilde{\Omega}_{j} \times\{0\}}\left(\widetilde{\Omega}_{j+1}\right)\right) \longrightarrow \cdots
\end{aligned}
$$

Here $\mathscr{B}_{\widetilde{\Omega}_{j} \times\{0\}}\left(\widetilde{\Omega}_{j+1}\right)$ denotes the space of hyperfunctions defined on $\widetilde{\Omega}_{j+1}$ and supported by $\widetilde{\Omega}_{j} \times\{0\}$. Note that $\widetilde{\Omega}_{j+1}-\tilde{\Omega}_{j} \times\{0\}=\widetilde{\Omega}_{j}^{+} \cup \widetilde{\Omega}_{j}^{-}$ and that $\widetilde{\Omega}_{j}^{+} \cap \widetilde{\Omega}_{j}^{-}=\varnothing$. Therefore $\operatorname{Ext}{ }^{i}\left(\tilde{M}_{j+1}, \mathscr{B}\left(\tilde{\Omega}_{j+1}-\widetilde{\Omega}_{j} \times\{0\}\right)\right) \cong$ $\operatorname{Ext}^{i}\left(\tilde{M}_{j+1}, \mathscr{B}\left(\widetilde{\Omega}_{j}^{+}\right)\right) \oplus \operatorname{Ext}^{i}\left(\tilde{M}_{j+1}, \mathscr{B}\left(\widetilde{\Omega}_{j}^{-}\right)\right)$.

Since $\tilde{M}_{n}$ is elliptic, Theorem 1 in Chapter VII $\S 11$ of Palamodov [9] claims

$$
\begin{aligned}
\operatorname{Ext}^{i}\left(\tilde{M}_{n},\right. & \left.\mathscr{B}\left(\widetilde{\Omega}_{n}\right)\right) \\
& =\operatorname{Ext}^{i}\left(\tilde{M}_{n}, \mathscr{B}\left(\widetilde{\Omega}_{n}^{+}\right)\right)=\operatorname{Ext}^{i}\left(\tilde{M}_{n}, \mathscr{B}\left(\widetilde{\Omega}_{n}^{-}\right)\right)=0
\end{aligned}
$$

for $i>2 n-(n+k+n)=k$. In fact, $\operatorname{Ext}^{i}\left(\tilde{M}_{n}, \mathscr{B}\left(\widetilde{\Omega}_{n}\right)\right)$ is isomorphic to $H^{i}\left(\widetilde{\Omega}_{n}, \mathscr{S}\right)$, where $\mathscr{S}$ denotes the hyperfunction solution sheaf of $\tilde{M}_{n}$. Since $\tilde{M}_{n}$ is elliptic, $\mathscr{S}$ is identified with the $C^{\infty}$-solution sheaf of $\tilde{M}_{n}$. Then it is again isomorphic to $\operatorname{Ext}^{i}\left(\tilde{M}_{n}, \mathscr{E}\left(\widetilde{\Omega}_{n}\right)\right)$, where $\mathscr{E}\left(\widetilde{\Omega}_{n}\right)$ denotes the sheaf of $C^{\infty}$-functions defined on $\widetilde{\Omega}_{n}$. On the other hand, the theorem of Palamodov [9] claims that $\operatorname{Ext}^{i}\left(\tilde{M}_{n}, \mathscr{E}\left(\widetilde{\Omega}_{n}\right)\right)=0$ for $i>2 n-(n$ $-k+n)$. Note that $\widetilde{\Omega}_{n}$ is an open set in $\mathbb{R}^{2 n}$ and that it is completely $(n-k+n)$-convex. The same argument applies also to $\operatorname{Ext}^{i}\left(\tilde{M}_{n}, \mathscr{B}\left(\widetilde{\Omega}_{n}^{+}\right)\right)$ and $\operatorname{Ext}^{i}\left(\tilde{M}_{n}, \mathscr{B}\left(\widetilde{\Omega}_{n}^{-}\right)\right)$. Thus (5) is proved. Therefore long exact sequence (4) implies that

$$
\operatorname{Ext}^{i}\left(\widetilde{M}_{n}, \mathscr{B} \widetilde{\Omega}_{n-1} \times\{0\}\left(\widetilde{\Omega}_{n}\right)\right)=0
$$

holds for $i>k+1$.

On the other hand Corollary 3.5.8 in Chapter II of Sato-KawaiKashiwara [11] shows that

$$
\operatorname{Ext}^{i}\left(\tilde{M}_{j}, \mathscr{B} \widetilde{\Omega}_{j-1} \times\{0\}\left(\widetilde{\Omega}_{j}\right)\right) \cong \operatorname{Ext}^{i-1}\left(\tilde{M}_{j-1}, \mathscr{B}\left(\widetilde{\Omega}_{j-1}\right)\right)
$$

holds for any $j \geqq 1$ and any $i$, since $\tilde{M}_{j-1}$ is a tangential system of $\tilde{M}_{j}$ induced onto $\tilde{\Omega}_{j-1}$. Here the symbol $\mathscr{B}$ in the left hand side of (7) denotes the sheaf of hyperfunctions in $(n+j)$-variables and the symbol $\mathscr{B}$ in the right hand side of (7) denotes the sheaf of hyperfunctions 
in $(n+j-1)$-variables. This remark should apply to all the formulas below. Note that (7) holds without the assumption of ellipticity of $\tilde{M}_{j}$. See Kashiwara-Kawai [4] Theorem 1.1 for the detailed proof of (7).

Combining (6) and (7), we immediately conclude that

$$
\operatorname{Ext}^{i}\left(\tilde{M}_{n-1}, \mathscr{B}\left(\tilde{\Omega}_{n-1}\right)\right)=0
$$

holds for $i>k$.

It is clear that the same argument applied to $\Omega \times I \underbrace{\times \cdots \times I}_{n-2} \times I^{ \pm} \times I$ instead of $\widetilde{\Omega}_{n}$ proves that

$$
\operatorname{Ext}^{i}\left(\tilde{M}_{n-1}, \mathscr{B}\left(\tilde{\Omega}_{n-1}^{ \pm}\right)\right)=0
$$

holds for $i>k$.

Therefore long exact sequence (4) proves that

$$
\operatorname{Ext}{ }^{i}\left(\tilde{M}_{n-1}, \mathscr{B} \widetilde{\Omega}_{n-2} \times\{0\}\left(\widetilde{\Omega}_{n-1}\right)\right)=0
$$

holds for $i>k+1$. Then (7) implies that

$$
\operatorname{Ext}^{i}\left(\tilde{M}_{n-2}, \mathscr{B}\left(\tilde{\Omega}_{n-2}\right)\right)=0
$$

holds for $i>k$.

Repeating these arguments successively, we finally arrive at the conclusion that

$$
\operatorname{Ext}^{i}\left(\tilde{M}_{0}, \mathscr{B}\left(\widetilde{\Omega}_{0}\right)\right)=0
$$

for $i>k$. Since $\tilde{M}_{0}=M$ and since $\widetilde{\Omega}_{0}=\Omega$ by the definition, we have proved the required vanishing theorem on completely $(n-k)$-convex set.

Remark. The result corresponding to Theorem 1 in the space of distributions is not known without the assumption of hypoellipticity of M. See Palamodov [9] Chapter VII §11.

Remark. Theorem 1 is a natural generalization of a theorem of Komatsu [7] on the existence of hyperfunction solutions of linear differential equations with constant coefficients on a convex open set. (See Theorem 3 of Komatsu [7].)

Now we introduce the notion of complete $k$-convexity for compact 
sets in $\mathbb{R}^{n}$ in order to discuss real analytic solutions of $M$.

Definition 2. A compact set $K \subset \mathbb{R}^{n}$ is said to be completely $k$-convex if and only if $K$ has a fundamental system of neighborhoods consisting of completely $k$-convex open sets.

Remark. Assume that there exists a $C^{2}$-function $h(x)$ defined in a neighborhood $U$ of $K$ which satisfies

(12) $K=\{x \in U ; h(x) \leqq 0\}$ is compact

and

(13) Hess $h(x)$ has at least $k$ positive eigenvalues in $U$.

Then $K$ is seen to be completely $k$-convex, since $U_{l}=\{x \in U ; h(x)$ $\left.<\frac{1}{l}\right\}(l \gg 1)$ is a completely $k$-convex set with norm function $\frac{1}{\frac{1}{l}-h(x)}$. In fact, choosing coordinate system $\left(x^{\prime}, x^{\prime \prime}\right)$ in $\mathbb{R}^{k} \times \mathbb{R}^{n-k}$ so that Hess $_{x^{\prime}} h(x)=\left(\frac{\partial^{2} h}{\partial x_{i} \partial x_{j}}\right)_{1 \leqq i, j \leqq k}$ is positive definite in an open set $\omega \subset U_{l}$, we see that $\operatorname{Hess}_{x^{\prime}} \frac{1}{\frac{1}{l}-h(x)}$ is positive definite in $\omega$, since it is equal to $\left(\frac{\frac{\partial^{2} h}{\partial x_{i} \partial x_{j}}}{\left(\frac{1}{l}-h(x)\right)^{2}}+\frac{2 \frac{\partial h}{\partial x_{i}} \frac{\partial h}{\partial x_{j}}}{\left(\frac{1}{l}-h(x)\right)^{3}}\right)_{1 \leqq i, j \leqq k}$. Note that $\sum_{i, j=1}^{k} \frac{\partial h}{\partial x_{i}} \frac{\partial h}{\partial x_{j}} \xi_{i} \xi_{j}$ $=\left(\sum_{i=1}^{k} \frac{\partial h}{\partial x_{i}} \xi_{i}\right)^{2} \geqq 0$ holds for any real vector $\left(\ddot{\xi}_{1}, \ldots, \xi_{k}\right)$.

Theorem 2. Let a compact set $K \subset \mathbb{R}^{n}$ be completely $(n-k)$ convex. Then, for any system $M$ of linear differential equations with constant coefficients defined on $\mathbb{R}^{n}$, we have

$$
\operatorname{Ext}^{i}(M, \mathscr{A}(K))=0
$$

for $i>k$. Here $\mathscr{A}(K)$ denotes the space of real analytic functions defined on $K$, i.e. $\mathscr{A}(K)=\varliminf_{l} \mathcal{O}\left(V_{l}\right)$, where $V_{l}$ runs through a fundamental system of Stein neighborhoods of $K$ in $\mathbb{C}^{n}$ and $\mathcal{O}\left(V_{l}\right)$ denotes the space of holomorphic functions defined on $V_{l}$.

Proof. First define the system $\tilde{M}_{n}$ on $\mathbb{R}^{2 n}$ as in the proof of 
Theorem 1. It is evident that

$$
\operatorname{Ext}^{i}\left(M, \mathcal{O}\left(V_{l}\right)\right)=\operatorname{Ext}^{i}\left(\tilde{M}_{n}, \mathscr{B}\left(V_{l}\right)\right)
$$

holds for every $i$, because each of the both hand sides is isomorphic to $H^{i}\left(V_{l}, \mathscr{S}\right)$, where $\mathscr{S}$ is the hyperfunction solution sheaf of $\tilde{M}_{n}$. Note that $\mathscr{S}$ is nothing but the holomorphic function solution sheaf of $M$ by the definition of $\tilde{M}_{n}$. Here we have used the fact that every linear differential equation with constant coefficients is solvable on an open convex set in the space of hyperfunctions and in the space of holomorphic functions. (See Komatsu [7].) In fact, in view of these solvability theorems combined with the vanishing of $H^{i}\left(V_{l}, \mathcal{O}\right)(i \geqq 1)$ and $H^{i}\left(V_{l}, \mathscr{B}\right)$ $(i \geqq 1)$, we can immediately apply the theory of spectral sequence to our case by making use of a locally finite covering $\mathscr{U}$ of $V_{l}$ consisting of open convex sets.

Since $\operatorname{Ext}^{i}(M, \mathscr{A}(K))=\varliminf_{l} \operatorname{Ext}^{i}\left(M, \mathcal{O}\left(V_{l}\right)\right)$ holds, it sufficies to prove that $\lim _{l} \operatorname{Ext}^{i}\left(\tilde{M}_{n}, \mathscr{B}\left(V_{l}\right)\right)=0$ holds for $i>k$. For this purpose, it suffices to show that $\varliminf_{l} \operatorname{Ext}^{i}\left(\tilde{M}_{n}, \mathscr{B}\left(\omega_{l}\right)\right)=0$ holds for $i>k$ for a fundamental system $\left\{\omega_{l}\right\}$ of open neighborhoods of $K$ in $\mathbb{R}^{2 n}$. By the assumption of complete $(n-k)$-convexity of $K$, we can take $U_{l} \times\left\{y \in \mathbb{R}^{n} ; \sum_{j=1}^{n}\left|y_{j}\right|\right.$ $\left.<\frac{1}{l}\right\} \subset \mathbb{R}^{n} \times \mathbb{R}^{n}$ as $\omega_{l}$, where $U_{l}$ is a completely $(n-k)$-convex open set in $\mathbb{R}^{n}$. Then $\omega_{l}$ is completely $(2 n-k)$-convex open set in $\mathbb{R}^{2 n}$, as is shown in the course of the proof of Theorem 1. Therefore Theorem 1 implies that $\operatorname{Ext}^{i}\left(\tilde{M}_{n}, \mathscr{B}\left(\omega_{l}\right)\right)=0$ for $i>k$. This immediately implies that $\varliminf_{l} \operatorname{Ext}^{i}\left(\tilde{M}_{n}, \mathscr{B}\left(\omega_{l}\right)\right)=0$ for $i>k$. This completes the proof of the theorem.

Remark. It is known (Kashiwara-Kawai [3]) that $\operatorname{Ext}^{i}(M, \mathscr{A}(\Omega)$ ) $=0$ holds for $i>k+1$ if $\Omega \subset \mathbb{R}^{n}$ is a completely $(n-k)$-convex open set. Here $\mathscr{A}(\Omega)$ denotes the space of real analytic functions defined on $\Omega$. This result was first proved by making use of the theory of boundary value problems for elliptic system of linear differential equations developed by Kashiwara-Kawai [4]. However, it is obvious that one can prove this result by making use of Theorem 2. Note that this result combined with Theorem 1 immediately proves that $\operatorname{Ext}^{i}(M$, $(\mathscr{B} / \mathscr{A})(\Omega)=0$ for $i>k$. Here $\mathscr{B} / \mathscr{A}$ denotes the quotient sheaf of $\mathscr{B}$ by 
sheaf $\mathscr{A}$ of real analytic functions. In contrast with the way of this argument, Kashiwara-Kawai [3] first establishes $\operatorname{Ext}^{i}(M,(\mathscr{B} / \mathscr{A})(\Omega))=0$ for $i>k$ to prove that $\operatorname{Ext}^{i}(M, \mathscr{A}(\Omega))=0$ for $i>k+1$.

By considering the adjoint system of $M$, Theorem 2 proves the vanishing theorem for relative cohomology groups as follows.

Theorem 3. Let a compact set $K \subset \mathbb{R}^{n}$ be completely $(n-k)$ convex. Assume that a system $M$ of linear differential equations with constant coefficients defined on $\mathbf{R}^{n}$ satisfies following condition (16):

$$
\operatorname{Ext}^{i}(M, A)=0 \quad \text { for } \quad i<d \text {. }
$$

Then we have

$$
\operatorname{Ext}^{i}\left(M, \mathscr{B}_{K}\left(\mathbb{R}^{n}\right)\right)=0
$$

for $i<d-k$. Here $\mathscr{B}_{K}\left(\mathbb{R}^{n}\right)$ denotes the space of hyperfunctions defined on $\mathbf{R}^{n}$ and supported by $K$.

Proof. First fix a free resolution of $M$ as follows:

$$
\cdots \longrightarrow A^{t_{2}} \stackrel{P_{1}^{\prime}}{\longrightarrow} A^{t_{1}} \stackrel{P_{0}^{\prime}}{\longrightarrow} A^{t_{0}} \longrightarrow M \longrightarrow 0 .
$$

Here $P_{j}^{\prime}$ is a transposed matrix of $P_{j}$, a matrix of polynomials of size $t_{j} \times t_{j+1}$. Condition (16) implies that the following dual sequence of (18) is exact.

$$
A^{t_{d}} \stackrel{P_{d-1}}{\longleftarrow} A^{t_{d-1}} \stackrel{P_{d-2}}{\longleftarrow} A^{t_{d-2}} \longleftarrow \cdots \longleftarrow A^{t_{2}} \stackrel{P_{1}}{\longleftarrow} A^{t_{1}} \stackrel{P_{0}}{\longleftarrow} A^{t_{0}} \longleftarrow 0 .
$$

If we define $A$-module $M^{\prime}$ by $A^{t_{a}} / P_{d-1} A^{t_{d-1}}$, then (19) gives rise to a free resolution of $M^{\prime}$. Therefore Theorem 2 implies that

$$
\operatorname{Ext}^{i}\left(M^{\prime}, \mathscr{A}(K)\right)=0
$$

holds for $i>k$. This is equivalent to saying that

$$
\operatorname{Ker}\left(P_{j-1}\left(D_{x}\right)^{\prime}:(\mathscr{A}(K))^{t_{j}} \longrightarrow(\mathscr{A}(K))^{t_{j-1}}\right)=P_{j}\left(D_{x}\right)^{\prime}\left(\mathscr{A}(K)^{t_{j+1}}\right)
$$

holds for $j<d-k$. Here $P_{j}\left(D_{x}\right)^{\prime}$ is a matrix of linear differential operator obtained by substituting a differential operator $\frac{\partial}{\partial x_{l}}$ to $\xi_{l}$. 
Since $\mathscr{A}(K)$ is naturally endowed with the topology of a dual Fréchet-Schwartz space and since its strong dual space is $\mathscr{B}_{K}\left(\mathbb{R}^{n}\right)$, we conclude by the closed range theorem that $P_{j}\left(D_{x}\right)\left(\left(\mathscr{B}_{K}\left(\mathbb{R}^{n}\right)\right)^{t_{j}}\right)$ is a closed subspace of $\left(\mathscr{B}_{K}\left(\mathbb{R}^{n}\right)\right)^{t_{j+1}}$ for $j<d-k$. (See Komatsu [6] and [8], for example.) Therefore (21) combined with the duality theorem of Serre (Komatsu [6]) asserts that

$$
\operatorname{Ker}\left(P_{j}\left(D_{x}\right):\left(\mathscr{B}_{K}\left(\mathbb{R}^{n}\right)\right)^{t_{j}} \longrightarrow\left(\mathscr{B}_{K}\left(\mathbb{R}^{n}\right)\right)^{t_{j+1}}\right)=P_{j-1}\left(D_{x}\right)\left(\left(\mathscr{B}_{K}\left(\mathbb{R}^{n}\right)\right)^{t_{j-1}}\right)
$$

holds for $j<d-k$. This is equivalent to saying that

$$
\operatorname{Ext}^{i}\left(M, \mathscr{B}_{K}\left(\mathbb{R}^{n}\right)\right)=0
$$

holds for $i<d-k$. This completes the proof of the theorem.

Having in mind the long exact sequence of relative cohomology groups, we now introduce the notion of complete $k$-concavity of an open subset $\Omega$ of $\mathbb{R}^{n}$.

Definition 3. An open set $\Omega \subset \mathbb{R}^{n}$ is said to be completely $k$ concave if and only if there exist a convex open set $U \subset \mathbb{R}^{n}$ and a completely $k$-convex compact set $K \subset U$ such that $\Omega=U-K$.

Remark. Assume that there exists a $C^{2}$-function $h(x)$ defined on a convex open set $U \subset \mathbb{R}^{n}$ which satisfies conditions (12) and (13). Then $\Omega=\{x \in U ; h(x)>0\}$ is completely $k$-concave. (See the remark after Definition 2.)

Theorem 4. Let $U$ be a convex open set in $\mathbb{R}^{n}$ and $\Omega=U-K$ be completely $(n-k)$-concave, i.e. $K$ be completely $(n-k)$-convex and compact in $U$. Let $M$ be a system of linear differential equations with constant coefficients defined on $\mathbb{R}^{n}$ which satisfies condition (16). Then we have

$$
\operatorname{Ext}^{i}(M, \mathscr{B}(\Omega))=0
$$

for $0<i<d-k-1$.

Proof. Since sheaf $\mathscr{B}$ of hyperfunctions is flabby (Sato [10]), we 
have the following long exact sequence:

$$
\begin{aligned}
\cdots \longrightarrow \operatorname{Ext}^{i}\left(M, \mathscr{B}_{K}(U)\right) \longrightarrow \operatorname{Ext}^{i}(M, \mathscr{B}(U)) \longrightarrow \operatorname{Ext}^{i}(M, \mathscr{B}(\Omega)) \\
\longrightarrow \operatorname{Ext}^{i+1}\left(M, \mathscr{B}_{K}(U)\right) \longrightarrow \operatorname{Ext}^{i+1}(M, \mathscr{B}(U)) \longrightarrow \cdots
\end{aligned}
$$

Since $U$ is convex, $\operatorname{Ext}^{i}(M, \mathscr{B}(U))=0$ for $i \geqq 1$. (Komatsu [7] Theorem 3.) Therefore we have

$$
\operatorname{Ext}^{i}(M, \mathscr{B}(\Omega)) \cong \operatorname{Ext}^{i+1}\left(M, \mathscr{B}_{K}(U)\right)
$$

for $i \geqq 1$. On the other hand, Theorem 3 implies under the assumptions of the theorem that

$$
\operatorname{Ext}^{i}\left(M, \mathscr{B}_{K}(U)\right)=0
$$

holds for $i<d-k$. Combining (26) and (27) we immediately see that

$$
\operatorname{Ext}^{i}(M, \mathscr{B}(\Omega))=0
$$

holds for $i<d-k-1$. This ends the proof of the theorem.

The exact sequence combined with Theorem 3 also proves the extendability of solutions of $M$ defined on $U-K$ even when $U$ is not necessarily convex, that is, we have the following theorem.

Theorem 5. Let a compact set $K \subset \mathbb{R}^{n}$ be completely $(n-k)$-convex. Let $U$ be an open neighborhood of $K$. Let $M$ be a system of linear differential equations with constant coefficients defined on $\mathbb{R}^{n}$ which satisfies condition (16) with $d>k+1$. Then for any hyperfunction solution $u(x)$ of $M$ defined on $U-K$ we can find a unique hyperfunction solution $\tilde{u}(x)$ of $M$ defined on $U$ so that it coincides with $u(x)$ on $U-K$.

Proof. Under the assumptions of the theorem, Theorem 3 implies that

$$
\operatorname{Ext}^{0}\left(M, \mathscr{B}_{K}(U)\right)=\operatorname{Ext}^{1}\left(M, \mathscr{B}_{K}(U)\right)=0
$$

Therefore long exact sequence (25) implies that

$$
\operatorname{Ext}^{0}(M, \mathscr{B}(U))=\operatorname{Ext}^{0}(M, \mathscr{B}(U-K))
$$


This is equivalent to saying that any hyperfunction solution $u(x)$ of $M$ defined on $U-K$ admits a unique extension $\tilde{u}(x)$ so that it is a hyperfunction solution of $M$ defined on $U$. This ends the proof of the theorem.

\section{References}

[1] Andreotti, A. and Grauert, H., Théorèmes de finititude pour la cohomologie des espaces complexes. Bull. Soc. Math. France, 90 (1962), 193-259.

[2] Ehrenpreis, L., A new proof and an extension of Hartos'g theorem. Bull. Amer. Math. Soc., 67 (1961), 507-509.

[3] Kashiwara, M. and Kawai, T., Applications of the theory of boundary value problems for elliptic system of linear differential equations. In preparation.

[4] - Theory and its applications of boundary value problems for elliptic system of linear differential equations. Proc. of Katata Symposium 1974, Sûrikaisekikenkyûsho Kokyâroku, No. 238, RIMS, Kyoto Univ. (1975), 1-59 (In Japanese).

[5] Kawai, T., Theorems on the finite-dimensionality of cohomology groups. IV. Proc. Japan Acad., 49 (1973), 655-658.

[6] Komatsu, H., Projective and injective limits of weakly compact sequences of locally convex spaces. J. Math. Soc. Japan, 19 (1967), 366-383.

[7] — Resolution by hyperfunctions of sheaves of solutions of differential equations with constant coefficients. Math. Ann., 176 (1968), 77-86.

[8] - Relative cohomology of sheaves of solutions of differential equations. Lecture Notes in Mathematics, No. 287. Springer-Verlag, Berlin-Heidelberg-New York (1973), 192-261.

[9] Palamodov, V. P., Linear Differential Operators with Constant Coefficients. Nauka, Moscow (1967) (In Russian). (Translated into English (Springer-Verlag, 1970) and into Japanese (Yoshioka, 1973)).

[10] Sato, M., Theory of hyperfunctions II. J. Fac. Sci. Univ. Tokyo, \& (1960), 387-437.

[11] Sato, M., Kawai, T. and Kashiwara, M., Microfunctions and pseudo-differential equations. Lecture Notes in Mathematics, No. 287. Springer-Verlag, BerlinHeidelberg-New York (1973), 265-529. 